\title{
Carbon - the first frontier of information processing
}

\author{
APOORVA PATEL \\ Centre for Theoretical Studies and Supercomputer Education and Research Centre, Indian Institute of Science, \\ Bangalore 560 012, India \\ (Fax,+91-80-3600106; Email, adpatel@cts.iisc.ernet.in)
}

\begin{abstract}
Information is often encoded as an aperiodic chain of building blocks. Modern digital computers use bits as the building blocks, but in general the choice of building blocks depends on the nature of the information to be encoded. What are the optimal building blocks to encode structural information? This can be analysed by substituting the operations of addition and multiplication of conventional arithmetic with translation and rotation. It is argued that at the molecular level, the best component for encoding discretized structural information is carbon. Living organisms discovered this billions of years ago, and used carbon as the back-bone for constructing proteins that function according to their structure. Structural analysis of polypeptide chains shows that an efficient and versatile structural language of 20 building blocks is needed to implement all the tasks carried out by proteins. Properties of amino acids indicate that the present triplet genetic code was preceded by a more primitive one, coding for 10 amino acids using two nucleotide bases.
\end{abstract}

[Patel A 2002 Carbon - the first frontier of information processing; J. Biosci. 27 207-218]

\section{Structural information}

It is a characteristic of living organisms to acquire information, interpret it and pass it on, often using it and refining it along the way. This information can be in various forms or languages. It can be genetic information passed on from the parent to the offspring, sensory information conveyed by the sense organ to the brain, linguistic information communicated by one being to another, or numerical data entered in a computer for later use. It is advantageous to process the information efficiently, and not in any haphazard manner. In case of living organisms, Darwinian selection during evolution can be considered the driving force for such optimization. In general, information processing is optimized following two guidelines: minimization of physical resources (time as well as space), and minimization of errors.

A striking feature of all the forms of information listed above is that the messages are represented as aperiodic chains of discrete building blocks. Such a representation, called digitization of the message, is commonplace due to its many advantages. Discretization makes it possible to correct errors arising from local disturbances, and so it is desirable even when the underlying physical variables, are continuous (e.g. voltages and currents in computers). It is also easier to handle several variables each spanning a small range than a single variable covering a large range. Any desired message can then be constructed by putting together the necessary number of the smaller range variables, while the instruction set required to manipulate each variable is substantially simplified. This simplification means that only a limited number of processes have to be physically implemented leading to high speed computation. An important question, therefore, is to figure out the best way of digitizing a message, i.e. what should be selected as the building blocks of the aperiodic chain.

The information contained in a message depends on the values and locations of the building blocks. Given a set of building blocks, Shannon quantified the information contained in a message as its entropy, i.e. a measure of the number of possible forms the message could have taken. This measure tells us that the information content

Keywords. Amino acid; aminoacyl-tRNA synthetase; computation; genetic code; information; lattice models; protein structure; quantum search; tetrahedral geometry 
of a message can be increased by eliminating correlations from it and making it more random. It also tells us that local errors in a message can be corrected by building long range correlations into it. But it does not tell us what building blocks are appropriate for a particular message. The choice of building blocks depends on the type of the information and not on the amount of information.

Information can be translated from one language into another by replacing one set of building blocks used to encode the information by another, e.g. textual information is stored in the computer in a binary form using the ascii code. Nonetheless, physical principles are involved in selecting different building blocks for different information processing tasks. For example, our electronic computers compute using electrical signals but store the results on the disk using magnetic signals; the former realization is suitable for quick processing while the latter is suitable for long term storage. In selection of building blocks with appropriate properties, the foremost practical criterion is that it should be easy to distinguish one building block from another. This simple criterion allows control over errors, and is often sufficient for a heuristic understanding of the number of building blocks of our languages. We use decimal system of numbers because we learnt to count with our fingers. The number of phonemes in our languages (i.e. vowels and consonants but not the tone) are determined by the number of distinct sounds our vocal chords can make. Computers and nervous systems use binary code because off/on states can be quickly decided with electrical signals. Genetic information is encoded using four nucleotide bases, perhaps because the quantum assembly algorithm is the optimal choice for replication at the molecular scale (Patel 2001).

Numerical representation of information is onedimensional and uses building blocks with an ordering amongst them (e.g. one is greater than zero). But these features may not be present in other types of information. For example, ordering is not required for letters of an alphabet, and representation of structural information requires higher dimensional building blocks. To find the building blocks most suitable to encode a particular type of information, one has to closely inspect the relation between the type of information and the physical properties and tasks associated with it.

The information in the genes for the synthesis of proteins is a clear-cut example of structural information. Living organisms generally do not have access to desired biomolecules in a readymade form. They first break down the ingested food into small building blocks, and then assemble the pieces in a precise manner to synthesize the desired biomolecules. The hereditary DNA is a one-dimensional read-only-memory in this process; the original DNA strand remains unchanged while the infor- mation contained in it is copied on the new strand that is assembled on top of it. The genes carry the blueprint of how to synthesize proteins by joining together their building blocks - the amino acids. The role of a protein in biochemical reactions is determined by its threedimensional shape and size, and the precise arrangement of chemical groups at its reaction sites. This threedimensional structural information of proteins is encoded as a one-dimensional chain of amino acids, with the interactions amongst the amino acids determining how the chain would bend and fold to produce protein structures. To investigate the details of this mechanism, it is natural to ask: what is the best way of encoding structural information? This is the question addressed in this work.

Many well-known properties of proteins and their relation to structural information are summarized in the Appendix. This material is provided only for quick reference, and those familiar with it can easily skip it. Every protein does not necessarily display all these properties, and there is considerable variation in the behaviour of different proteins (e.g. between small and large proteins). On the other hand, it has to be emphasized that an efficient and yet versatile language must be capable of incorporating all the desired features, even though specific instances of the language may not display every possible feature. The ideal language for proteins, therefore, must have the capability to: (i) fold linear chains into three-dimensional structures and also unfold them, (ii) form three-dimensional structures of different shapes and sizes, (iii) include different chemical groups as part of the amino acids, and (iv) show chiral behaviour.

Any structural transformation of a rigid body can be described in terms of two basic operations, translations and rotations. The set of all rigid body translations and rotations forms the well-known Galilean group, which has been studied in detail by physicists. To construct the building blocks of structural information, we have to discretize this continuous group and yet maintain its features required to encode information.

We can compare translations and rotations to the fundamental operations of arithmetic - addition and multiplication. While addition is nothing but translation along the real line, multiplication is quite different from rotation. Rotations in our three-dimensional space are not commutative and that is of crucial importance in representing structural information. (The group of three-dimensional rotations is $S U(2)$, which can be represented using Pauli matrices or quaternions.) The building blocks of numerical information are elements of $Z_{n}$, the group of integers modulo $n$, and the cyclic nature of this group represents the order amongst the building blocks. The building blocks of structural information need to have characteristics of rigid bodies, i.e. specific size and orientation in 
three-dimensional space. To find them we have to look for a finite non-commutative group. In addition, to address the question of protein structure, we should look for transformations that take place at the atomic scale.

Translations are easily discretized, as uniformly spaced units along a polymer chain. The atomic structure of matter provides a natural unit for translation - the physical size of the building blocks. Indeed, the amino acids making up proteins differ from each other in terms of their side chemical groups, while their components along the chain are identical. Any translation can be built up from the elementary operations of addition of a building block, deletion of a building block and exchange of two adjacent building blocks.

Rotations are more complicated to discretize. A reasonable criterion is to demand, on the basis of symmetry, that the allowed states be all equivalent and equidistant from each other. The largest set of such states can then provide an approximate basis for the rotation group, and the following properties are quickly discovered:

- In our three-dimensional world, the largest number of equivalent and equidistant states is four. They correspond to the corners of a regular tetrahedron. One can go from any one to any other with equal ease, in just one step.

- A tetrahedron is the smallest polyhedron. It is the simplest structure that can implement non-commutative features of three-dimensional rotations. (In general, the simplest unit for tiling a $d$-dimensional space is a simplex with $(d+1)$ vertices. It is often convenient to construct a $d$-dimensional space as a Cartesian product of $d$ onedimensional spaces, but the simplex is a much more flexible unit than a hypercube.)

- To be able to specify the three-dimensional orientation unambiguously, the building blocks should have the capability to include a chiral centre. Tetrahedral geometry allows that.

- If quantum dynamics is involved, then the states should also be mutually orthogonal, so that they form a basis for the Hilbert space. The tetrahedral quantum states are mutually orthogonal; they can be obtained from the lowest two spherical harmonics, $l=0$ and $l=1$. $(l=0,1$ form the minimal basis set for specifying orientations in three-dimensional space. They are the smallest two representations of the group of proper rotations, and any other representation can be constructed from them by tensor products.) Using $s p^{3}$-hybridization of atomic orbitals, these states can be denoted as:

$$
\left(\begin{array}{l}
\alpha \\
\beta \\
\gamma \\
\delta
\end{array}\right)=\left(\begin{array}{rrrr}
1 / 2 & 1 / 2 & 1 / 2 & 1 / 2 \\
1 / 2 & 1 / 2 & -1 / 2 & -1 / 2 \\
1 / 2 & -1 / 2 & 1 / 2 & -1 / 2 \\
1 / 2 & -1 / 2 & -1 / 2 & 1 / 2
\end{array}\right)\left(\begin{array}{c}
s \\
p_{x} \\
p_{y} \\
p_{z}
\end{array}\right)
$$

The high symmetry of this unitary transformation (all elements equal, only signs differ) is related to the equivalence of the four states.

- Four is also the largest number of states which can be uniquely identified by a single yes/no question in a quantum search algorithm (Grover 1997).

\section{Tetrahedral geometry}

The outstanding example of an element with such states is carbon. Moreover,

- Carbon has the capability to form aperiodic chains, where different side chemical groups hang on to a backbone. This capability is a must for encoding information. Silicon also possesses the same tetrahedral states, and is much more abundant, but it preferentially forms periodic chains (i.e. regular crystals).

- If the logic above is repeated in the case of twodimensional rotations, it leads to three equivalent states located at the corners of an equilateral triangle. Carbon has the capability to form these states as well, by $s p^{2}$ hybridization of its atomic orbitals.

- Carbon is the most important structural element forming the back-bone of biomolecules. Darwinian selection in evolution can be expected to have picked the best building blocks out of the available resources.

With all these pieces fitting together, let us look at the tetrahedral group in some detail. The tetrahedral group is isomorphic to the permutation group of four objects. It has 24 elements, which can be factored into a group of 12 proper rotations (or even permutations) and reflection (or parity). The 24 element and 12 element groups are denoted as $T_{d}$ and $T$ respectively.

A regular tetrahedron can be formed by joining alternate corners of a cube. The centres of the tetrahedron and cube then coincide, and this embedding is convenient for three-dimensional structural analysis of a chain with tetrahedral angles. The 12 proper rotations are decomposed into the identity operation, rotations around 3 -fold axes and rotations around 2-fold axes. There are four 3fold axes, each joining the centre of the tetrahedron with a vertex; $+120^{\circ}$ and $-120^{\circ}$ rotations around these axes belong to different equivalence classes. There are three 2-fold axes, each passing through the centre of the tetrahedron and midpoints of its non-intersecting edges (equivalently passing through the centres of opposite faces of the embedding cube).

For a carbon atom located at the centre of the tetrahedron, rotations around 3-fold axes correspond to rotations around its bonds. These single bonds are easy to rotate and give rise to different conformations of organic molecules. In a polypeptide chain, the orientations that can be achieved by rotations around the bonds of the $C_{\alpha}$ 
atoms are described by the Ramachandran map. As shown in figure 1 , the rotation angles are not uniformly populated, but prefer to be in several discrete locations. As the stars in the plot show, discrediting the angles in steps of $120^{\circ}$ is not a bad starting point.

The 2-fold rotation axes bisect the bond angles. If a double bond is viewed as a deformation in which two tetrahedral bonds are merged together, then the double bond lies along the 2 -fold rotation axis. $180^{\circ}$ rotation about this axis corresponds to a transition between "trans" and "cis" forms. Most of the peptide bonds have the "trans" configuration. But occasional transitions to the "cis" form do occur, and they are important for introducing sharp bends in the chain.

The parity transformation flips chirality of a structure, which is of special significance for many biological molecules. Chirality flip is an allowed quantum transformation, e.g. the $\mathrm{NH}_{3}$ molecule flips back and forth between configurations where the nitrogen atom is above and below the plane of three hydrogen atoms. But chirality flip becomes more difficult as the molecular size increases, and all the amino acids used as building blocks of proteins are known to be L-type (except for achiral glycine). Thus reflections are more difficult to implement than proper rotations, and can be ignored as far as the structural analysis of proteins is concerned.

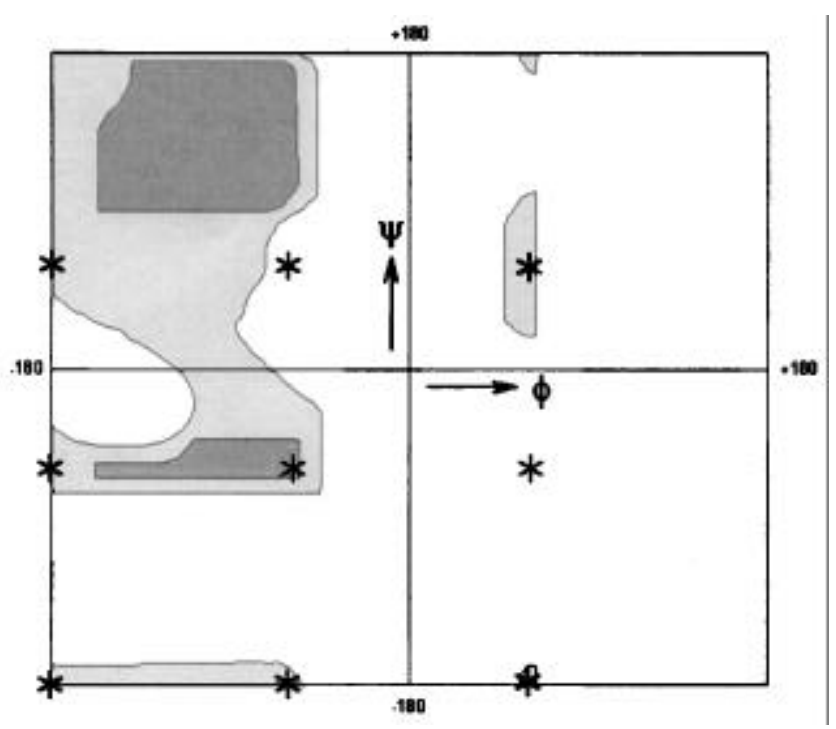

Figure 1. The Ramachandran map for chiral L-type amino acids, displaying the permitted rotation angles for the $\mathrm{C}_{\alpha}$ bonds in polypeptide chains (Ramachandran 1963). The angles $\phi$ and $\psi$ are periodic. In the approximation that embeds the polypeptide chain on a diamond lattice in the "trans" configuration, only nine discrete possibilities exist for the rotation angles. These are marked by stars on the same plot; they are uniformly separated by $120^{\circ}$ steps.

\section{Packing three-dimensional information}

Multi-dimensional structural information can be encoded in several different ways. The complete information can be expressed directly, as in holograms (3-dim) and movie projections (2-dim). Or it can be arranged as an ordered set of lower dimensional segments, as in CT-scan (stack of parallel planes covering a 3-dim object) and television monitors (set of lines covering a 2-dim picture). The choice depends on whether the physical means that convey the information are extended or local. When mechanisms exist to look at the whole object in one go (e.g. with a wide beam of light), the complete information can be addressed directly. When only one part of the object can be considered at a time (e.g. with a narrow beam of electrons), it is more convenient to arrange the information as a sequence of small segments. When the building blocks themselves have to convey the information, the latter format is the obvious choice; multi-dimensional arrays are stored as folded sequences in computers and proteins are assembled as folded polypeptide chains.

It is possible to assemble arbitrary structures by repetitive arrangement of a single and small enough building block. For example, a crystal can be carved into the desired shape, and it is sufficient to describe the details of the surface (and not the contents of the full volume) for that purpose. A crystal can also form rapidly, since it can grow from a seed in all directions. But the preferred shape of the crystal remains that of the building block. To assemble arbitrary shapes using a crystalline arrangement, another agency is needed to tell the crystal surface to stop growing after it has reached the desired position as well as to put the reactive chemical groups at specific locations; the building blocks themselves cannot carry those instructions. Thus crystal growth is convenient for making regular patterns, but it is not a good choice for assembling irregular shapes.

The highly non-trivial task of specifying an irregular structure can be more easily accomplished by an aperiodic folded chain of building blocks. Then the building blocks themselves carry preferences for specific orientations at each step. Although such a chain grows slowly, it does not need help from an external agency to achieve its desired shape. This property is a must at the lowest level of information processing - the message has to carry its own interpretation in terms of its physical properties; no other interpreter is available ${ }^{1}$. With a chain

\footnotetext{
${ }^{1}$ In case of computers, compilers and operating systems provide the abstract interpretation for high level information processing. But at the lowest level of machine code, the interpretation is built into the design of the physical components, i.e. in their responses to applied voltages and currents.
} 
that knows how to fold itself, the problem of specifying the three-dimensional structural detail is simplified to that of constructing the appropriate one-dimensional chain. It is far easier for an external agency to synthesize aperiodic one-dimensional chains than irregular three-dimensional structures. Proteins do not have regular shapes; they need all their grooves and cavities (i.e. structural defects) for their function, and how they fold is decided by their building blocks joined in a polypeptide chain. Another physical reason why proteins have to be polypeptide chains that can fold and unfold again is that many proteins have to cross membranes and cell walls to carry out their tasks. A bulky shape would require a big hole in the barrier to be crossed, through which many other molecules could also leak. But proteins unfold to their chain form, slip through a small hole in the barrier, and then fold again to their native form.

Even after picking a folded chain structure, more specifications are needed to find the desired building blocks. The chain can be uniformly flexible like a piece of string, or it can be made of stiff segments alternating with flexible joints like a chain of metal rings. If all the segments of a chain are flexible, then it has to be fully tied from all directions to be held in place. Otherwise the structure can crumple and collapse. Carbon forms many structures with fully saturated bonds, but a completely tied threedimensional form requires rather precise folding and cannot accommodate aperiodic building blocks easily. Moreover, for a chain to have the capacity to fold and unfold again, the side bonds holding the folds in the chain must be weaker than the bonds along the backbone. For example, diamond is the hardest material, but it is a periodic structure and cannot be folded and unfolded again easily. The polyethylene back-bone [i.e. $\left(-\mathrm{CH}_{2}-\right)_{n}$ ] can accommodate aperiodic side groups, but it is too flexible. Given that the side group interactions are necessarily weak, structural stability can be enhanced by making the back-bone stiffer, e.g. by replacing some of the single bonds with double bonds that cannot rotate. Polypeptide chains are of this type; they have weak side group interactions, and the increased stiffness of their non-rotatable peptide bonds helps in maintaining the shape of the protein.

Figure 2 a shows a back-bone with alternating single and double bonds. This is the structure of polyacetylene, and an aperiodic chain can be constructed by replacing the side $-\mathrm{H}$ by other chemical groups (e.g. $-\mathrm{CH}_{3}$ ). The trouble with this structure is that the $\pi$-electrons involved in double bonds prefer to lower their energy by spilling over into neighbouring bonds. This resonance phenomenon gives a double bond character to all the bonds (the actual bond properties are somewhere in between a single and a double bond), and makes the whole back-bone planar. A planar back-bone is no good for constructing three-dimensional structures. The double bonds can be shifted to the side groups to reduce the spill over of $\pi$ electrons, as illustrated in figure $2 b$, but the resultant structure is still planar. The next possibility for the backbone configuration, which allows stiff segments with flexible joints, is to alternate one double bond with two single bonds. This is the structure of polypeptide chains, as shown in figure $2 \mathrm{c}$. The stiff $\mathrm{C}-\mathrm{N}$ peptide bond is created by $\pi$-electrons spilling over from the $\mathrm{C}=\mathrm{O}$ double bond; inclusion of nitrogen atoms in the chain ensures that the $\pi$-electrons spill over only on one side and not the other. The rotatable single bonds of $\mathrm{C}_{\alpha}$ atoms permit construction of three-dimensional structures.

The configurations of amino acids that polymerize to form the polypeptide chain are shown in figure 3 . The presence of acidic $-\mathrm{COOH}$ and basic $-\mathrm{NH}_{2}$ groups in all amino acids provides a convenient way to join them in polypeptide chains by acid-base neutralization.

\section{Elementary building blocks}

Having analysed the merits of a polypeptide back-bone structure, we now look at the three-dimensional geometry of a polypeptide chain, but with the simplifying assumptions that all the links in the chain are of equal length and all the tetrahedral angles are of equal value $\left(2 \tan ^{-1}\right.$ $\left.(\sqrt{ } 2) \approx 109 \cdot 5^{\circ}\right)$. With these assumptions, the folded chain lies on a diamond lattice. Although the real peptide bond is planar with angles close to $120^{\circ}$, it can be fitted reasonably well on the diamond lattice in the "trans"<smiles>C=CC=C</smiles>

(a)<smiles>CC(=O)C(=O)C(C)=O</smiles>

(b)<smiles>[R]C([R])C(=O)N([R])NC([R])([R])C(N)=O</smiles>

(c)

Figure 2. Different possibilities for polymer chains with carbon back-bone: $(\mathbf{a})(\mathrm{CH})_{n},(\mathbf{b})(\mathrm{CO})_{n}$, (c) polypeptide chain. 
configuration (see figure 4). The rare "cis" configuration, takes the chain out of the diamond lattice. Let us first keep the "cis" configuration aside, and consider the chain in the "trans" configuration only. In a real polypeptide chain, variations from equal bond lengths and equal angles are within $\pm 10 \%$, and I will analyse the above described simplified version using the conventional polypeptide chain nomenclature.

The diamond lattice is a face-centred cubic lattice with a two-point basis. Let this basis of lattice points be $(0,0,0)$ and $(1 / 4,1 / 4,1 / 4)$ in units of the unit cell. Then the bond directions of the diamond lattice are (these are the last three columns of the matrix in eq.(1)):

$$
\begin{aligned}
& e_{1}=(+1 / 4,+1 / 4,+1 / 4) \\
& e_{2}=(+1 / 4,-1 / 4,-1 / 4) \\
& e_{3}=(-1 / 4,+1 / 4,-1 / 4) \\
& e_{4}=(-1 / 4,-1 / 4,+1 / 4) .
\end{aligned}
$$

These directions refer to the lattice point at the origin, and thereafter the bond directions at neighbouring points are opposite in sign.

We can enumerate all possible configurations of the polypeptide chain, by specifying for every peptide bond the location of the next peptide bond in the chain. Let the reference peptide bond $(\mathrm{C}-\mathrm{N})$ be along $e_{1}$ from the origin. The chain prior to this peptide bond is already synthesized, so without loss of generality let the location of the $\mathrm{C}_{\alpha}$ preceding the reference peptide bond be $e_{2}$. In the "trans" configuration, the $\mathrm{N}-\mathrm{C}_{\alpha}$ and the $\mathrm{C}_{\alpha}-\mathrm{C}$ bonds are parallel, so the location of the $\mathrm{C}_{\alpha}$ following the reference peptide bond is fixed as $e_{1}-e_{2}$. (The sequence $\mathrm{C}_{\alpha}-\mathrm{C}-\mathrm{N}-\mathrm{C}_{\alpha}$ fixes the plane of the peptide bond.)

There are three possible locations for the next $\mathrm{C}$ : $e_{1}-e_{2}+e_{1}, e_{1}-e_{2}+e_{3}$ and $e_{1}-e_{2}+e_{4}$. From each of these three locations, the next peptide bond can proceed along three possible directions, excluding the already occupied $\mathrm{C}_{\alpha}-\mathrm{C}$ direction. The $\mathrm{C}_{\alpha}-\mathrm{C}$ direction and the next peptide bond direction fix the plane of the next peptide bond. Thus on a diamond lattice, given a peptide bond plane, there are 9 possible positions for the next peptide bond plane.<smiles>NCC(=O)[O-]</smiles>

(a)

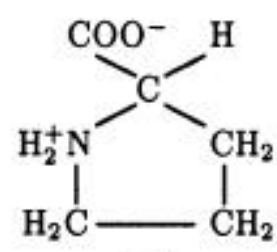

(b)<smiles>[R]C(N)C(=O)[O-]</smiles>

(c)
Figure 3. Amino acid configurations (in their ionized forms): (a) glycine, (b) proline, (c) all the rest.
These 9 orientations are all geometrically equivalent they just reflect the 3-fold rotational symmetry around tetrahedral bonds of the $\mathrm{C}_{\alpha}$ atom. When $\mathrm{C}_{\alpha}-\mathrm{N}$ bond is held in position, there are three equivalent choices of $\phi$, and when $\mathrm{C}_{\alpha}-\mathrm{C}$ bond is held in position, there are three equivalent choices of $\psi$.

For each of the polypeptide back-bone configuration described above, there are two remaining directions for other groups to attach to the $\mathrm{C}_{\alpha}$ atom. One direction is attached to the $R$-group of the amino acid, while the other to a hydrogen atom. There are two arrangements possible, and they correspond to opposite chirality. Detailed model-building studies have shown that all the $R$-groups in a polypeptide chain must be of the same stereoisomer for the stability of regular secondary structures (e.g. $\alpha$ helices and $\beta$-sheets). All the amino acids naturally occurring in proteins are L-type. Altogether, therefore, there remain 9 possible ways of adding a new L-type amino acid to an existing polypeptide chain. (Instead of an $R$-group, glycine has two hydrogen atoms attached to the $\mathrm{C}_{\alpha}$ atom. That makes glycine achiral, but the number of attachment possibilities for the $\mathrm{C}_{\alpha}$ atom remains one.)

The Ramachandran map shown in figure 1 is constructed using the bond lengths and angles in an actual polypeptide chain. The nine points corresponding to the "trans" configuration discrete chain are marked as stars on the same plot. It is easily seen that the discrete approximation is not too far off reality, even though it cannot describe all the details of the $\phi-\psi$ angular distribution. Actually, the plot in figure 1 does not include glycine and proline; their structural preferences are somewhat different. The region around $\left(\phi=60^{\circ}, \psi=\right.$ $\left.-60^{\circ}\right)$ is not occupied in the Ramachandran map because of steric conflict between the side chain $R$-group and the atoms in the polypeptide back-bone. Glycine with no side chain does not have this conflict and can occupy this region - its Ramachandran map has inversion symmetry. In case of proline, the rigid imino ring does not allow the $\mathrm{N}-\mathrm{C}_{\alpha}$ bond to rotate, and $\phi$ is constrained to be around $-60^{\circ}$. In case of a real polypeptide chain, embedding it on the diamond lattice will distort its shape; the extent of distortion will then be a measure of usefulness of the discretized description. (The peptide bond is a little shorter than the single bonds and its bond angles of $120^{\circ}$ are somewhat wider than the tetrahedral angle. These two deviations tend to compensate for each other to some extent.)

Now we can look at the "cis" configuration of the peptide bond. It is obtained from the "trans" configuration by rotating the $\mathrm{N}-\mathrm{C}_{\alpha}$ bond by $180^{\circ}$ around the peptide bond axis (see figure 4 ). With the peptide bond along $e_{1}$ and the preceding $\mathrm{C}_{\alpha}-\mathrm{C}$ bond along $-e_{2}$, the "cis" configuration $\mathrm{N}-\mathrm{C}_{\alpha}$ bond is along $\frac{2}{3} e_{1}+e_{2}$. This orientation does not fit in the face-centred cubic diamond 
<smiles>CCCC(=O)NCC</smiles>

(a)<smiles>CCC(=O)NC=O</smiles>

(b)
Figure 4. Peptide bond configurations: (a) trans, (b) cis.

lattice, but it can be fitted in the hexagonal diamond lattice $^{2}$ with the hexagonal symmetry axis along $e_{1}$. It is well-known that the three-dimensional closest packing of spheres can be viewed as a stack of two-dimensional layers. There are three possible positions for the layers, and each layer has to be displaced relative to the ones on its either side. There are, therefore, two distinct ways to add a new layer onto an existing stack. The facecentred cubic lattice corresponds to the layer sequence ... ABCABCABC. . ., the hexagonal lattice corresponds to the sequence ... ABABAB ...., and random sequences are also possible. An insertion of a "cis" peptide bond in an otherwise "trans" peptide chain corresponds to a flip in the layer sequence of the type ... ABCABCBACBA .... This flip has no effect on the 9 possibilities for the subsequent rotation angles $\phi$ and $\psi$, and further elongation of the polypeptide chain. Thus we can count the trans-cis transformation as one more elementary structural operation.

The 10 operations described above exhaust the "elementary logic gates" for the polypeptide chain embedded on a diamond lattice, i.e. by implementing these 10 operations one can fold the polypeptide chain on a diamond lattice in any desired configuration. Long distance connections (disulfide and hydrogen bonds) are important for structural stability of the polypeptide chain, but they do not give rise to new configurational possibilities.

\section{Putting things together}

There is no clear association of any amino acid with the 9 discrete points in the Ramachandran map. Even the most

\footnotetext{
${ }^{2}$ Carbon can also form a hexagonal diamond lattice, with the same tetravalent bonds and density as the face-centred cubic diamond lattice. Such hexagonal diamond crystals do not occur terrestrially, but they have been found in meteorites and have been synthesized in laboratory.
}

rigid proline occurs in different orientations, and though only glycine can occupy the region around $\left(\phi=60^{\circ}, \psi=\right.$ $-60^{\circ}$ ) it occurs in other orientations too. Indeed, just the composition of a particular amino acid does not decide its configuration in the polypeptide chain; rather the overall interactions of its $R$-group with those that precede it and those that follow it fix the configuration. When the orientation of the middle amino acid depends on the amino acids that precede and follow it, the structural code is necessarily an overlapping one. Even with an overlapping structural code, every time an amino acid is added to the polypeptide chain, the orientation of one amino acid gets decided. So a maximally overlapping efficient code needs at least 9 amino acids (may be 10 to include trans-cis transformation) to construct a polypeptide chain of arbitrary configuration ${ }^{3}$. The $R$-group properties of amino acids have been studied in detail: polar and non-polar, positive and negative charge, straight chains and rings, short and long chains, and so on. Still which sequence of amino acids will lead to which conformation of the chain is an exercise in coding that has not been solved yet.

At this stage, it is instructive to observe that the 20 amino acids are divided into two classes of 10 each, according to the properties of their aminoacyl-tRNA synthetases (Eriani et al 1990; Arnez and Moras 1997; Lewin 2000). The two classes of synthetases totally differ from each other in their active sites and in how they attach amino acids to the tRNA molecules. The lack of any apparent relationship between the two classes of synthetases has led to the conjecture that the two classes evolved independently, and early form of life could have existed with proteins made up of only 10 amino acids of one type or the other. A closer inspection of the $R$-group properties of amino acids in the two classes reveals that each property (polar, non-polar, ring/aromatic, positive and negative charge) is equally divided amongst the two classes, as shown in table 1 . Not only that, but the heavier amino acids with each property belong to class I, while the lighter ones belong to class II. This division of amino acids according to the length of their side chains

\footnotetext{
${ }^{3}$ Information is localized in individual building blocks in a strictly local code (e.g. our system of writing numbers), but it is not so in an overlapping code (e.g. pronunciation of vowels in English words often depends on the neighbouring letters). As long as the total information content of the message remains the same, one can map the two codes into each other by changing variables. When both the codes are efficient, the total length of the message and the total information content remain the same, and the number of building blocks required cannot change. One can reduce the number of building blocks at the expense of increasing the length of the message, but that would not make the best use of available resources.
} 
has unambiguous structural significance. The diamond lattice structure is quite loosely packed with many cavities of different sizes. The use of long side chains to fill up big cavities and short side chains to fill up small ones can produce a dense compact structure, and proteins indeed are tightly packed close to the maximum packing fraction. Thus we arrive at a structural explanation for the 20 amino acids as building blocks of proteins, a factor of 10 for conformations of the polypeptide back-bone and a factor of 2 for the length of the $R$-group. (It can be noted that each class contains one special amino acid involved in tasks beyond the 9 folding possibilities on the diamond lattice. Proline is the special amino acid in class II, involved in trans-cis transformations, while cysteine is the special amino acid in class I, involved in tying together far separated regions of the polypeptide chain.)

A look at the optimal solutions of the quantum search algorithm (Grover 1997) brings out another interesting feature. Living organisms form DNA and polypeptide chains by joining their building blocks together in sequential assembly line operations. The templates for

Table 1. Properties of the amino acids depend on their side chain $R$-groups. Larger molecular weights indicate longer side chains. The 20 amino acids naturally occurring in proteins have been divided into two classes of 10 each, depending on the properties of aminoacyl-tRNA synthetases that bind the amino acids to tRNA. These classes divide amino acids with each $R$ group property equally, the longer side chains correspond to class I and the shorter ones correspond to class II.

\begin{tabular}{llcc}
\hline Amino acid & \multicolumn{1}{c}{$\begin{array}{c}R \text {-group } \\
\text { property }\end{array}$} & $\begin{array}{c}\text { Mol. } \\
\text { wt. }\end{array}$ & Class \\
\hline Gly (glycine) & Non-polar & 75 & II \\
Ala (alanine) & Non-polar & 89 & II \\
Pro (proline) & Non-polar & 115 & II \\
Val (valine) & Non-polar & 117 & I \\
Leu (leucine) & Non-polar & 131 & I \\
Ile (isoleucine) & Non-polar & 131 & I \\
Ser (serine) & Polar & 105 & II \\
Thr (threonine) & Polar & 119 & II \\
Asn (asparagine) & Polar & 132 & II \\
Cys (cysteine) & Polar & 121 & I \\
Met (methionine) & Polar & 149 & I \\
Gln (glutamine) & Polar & 146 & I \\
Asp (aspartate) & Negative charge & 133 & II \\
Glu (glutamate) & Negative charge & 147 & I \\
Lys (lysine) & Positive charge & 146 & II \\
Arg (arginine) & Positive charge & 174 & I \\
His (histidine) & Ring/aromatic & 155 & II \\
Phe (phenylalanine) & Ring/aromatic & 165 & II \\
Tyr (tyrosine) & Ring/aromatic & 181 & I \\
Trp (tryptophan) & Ring/aromatic & 204 & I \\
\hline
\end{tabular}

Some specific properties not explicit in the table are: asparagine is a shorter side chain version of glutamine, histidine has an $R$-group with a small positive charge but it is close to being neutral, and both the sulphur containing amino acids (cysteine and methionine) belong to class I. the assembly, hereditary DNA and mRNA, are preexisting objects in these processes. The building blocks are available as a random collection, and the correct ones are selected by ensuring that they form appropriate molecular bonds with the templates. Molecular bonds are binary questions - they either form or do not form. The optimization criterion is to select the correct building block from the random collection by asking the minimum number of questions. With binary questions, the best classical search algorithm is a binary search, but the best quantum search algorithm is different. Identification of the nucleotide base-pairing with a binary quantum question provided two significant results for genetic information processing (Patel 2000): the largest number of items that can be distinguished by one quantum question is 4 , and by three quantum questions is $20 \cdot 2$. These numbers match the number of letters in DNA and protein alphabets, and the triplet code translating between them. The same algorithm also predicts that the largest number of items that can be distinguished by two quantum questions is $10 \cdot 5$. (The non-integer number of items means that the algorithm has an intrinsic error. In the case of two queries and 10 items, the error rate is about 1 part in 1000.) A two nucleotide base code is thus optimal for distinguishing 10 amino acids.

The experimentally observed wobble rules are consistent with the idea that an earlier genetic code used only two nucleotide bases of every codon and synthesized a smaller number of amino acids (Crick 1966). Another feature supporting this idea is that in the present genetic code similar codons code for amino acids with similar $R$ group properties. It is therefore possible that the third codon entered the present genetic code as a class label (classical and not quantum), when two independent codes corresponding to long and short $R$-groups merged together during the course of evolution. Such symbiosis is not uncommon - there is evidence that the cellular organelles mitochondria and chloroplasts, with their own genetic material, first developed independently and were later incorporated in ancestral cells with eukaryotic nuclei.

Combining these arguments, we can now construct a possible scenario of how the present genetic code arose from a more primitive one. Since the discovery of the genetic code, many attempts have been made to find its simpler predecessors (see for instance, Crick 1968; Kolaskar and Ramabrahmam 1982; Ikehara 2002). The present genetic code is too complex to have arisen at one instance, and all the simpler predecessors use fewer nucleotide bases and fewer amino acids. The most important criterion in these attempts is that continuity has to be maintained in evolution - a drastic change will not permit the organism to survive. The changes therefore have to combine many small steps, each small change in the code providing a certain advantage in functionality. The scenario 
suggested by the preceding arguments is similar to what Crick proposed many years ago (Crick 1968):

(i) The primitive code was a triplet due to some unidentified reasons. The first two letters coded for 10 amino acids, while the third letter was a non-coding separation mark. The individual genes were separate and not joined together, and so START and STOP signals were not needed.

(ii) This primitive code synthesized the simpler class II amino acids. The information about how the polypeptide chain twists and turns at each step was incorporated in this code. The short side chains of class II amino acids, however, could not completely fill all the cavities in the three-dimensional protein structure.

(iii) The longer class I amino acids replaced the short ones of similar property at a later stage, wherever big cavities existed. This filling up of cavities increased the structural stability of proteins.

(iv) The third letter was put into use as a double-valued classical label for the amino acid class. That allowed coding for 20 amino acids.

(v) Further optimization of the code occurred with some juggling of codons, since 20 amino acids can be coded either by one classical and two quantum queries or by three quantum queries. Also, many genes joined together and START and STOP signals were inserted.

(vi) Similar codons for similar amino acids and the wobble rules are relics of the doubling of the genetic code, indicative of the past but no longer perfectly realized.

In the absence of any knowledge of the doublet code, it is not possible to pin-point this scenario any further, and variations can be imagined.

Further progress along this direction requires solutions of two puzzles. First, as already pointed out above, we need to identify which amino acid subsequence corresponds to which structural building block. There is no clear criterion regarding how long the amino acid subsequence should be before it assumes a definite shape; may be interactions of an amino acid with two preceding ones and two following ones is a good enough beginning. The protein structure data accumulated in databases should help in such an analysis. Second, we need to guess the doublet code assignments from the known triplet code. This has already been studied to some extent within the context of the wobble rules, but it should be investigated in more detail keeping the constraints of the aminoacyl-tRNA synthetase classes in mind.

\section{Summary and outlook}

I have looked at the structure of proteins from an information theory point of view. The emphasis is on the three-dimensional structure of the end-product, i.e. how should the segments of a polypeptide chain be chosen so that it folds into the required shape. The means used to achieve that end are secondary, i.e. which amino acids should be chosen so that the interactions amongst their $R$ groups make the polypeptide chain fold in the required manner. This emphasis is in sharp contrast with the conventional approach to the protein folding problem, i.e. find the three-dimensional structure of the protein, given the sequence of amino acids and the interactions of their $R$-groups. The conventional approach requires finding the lowest energy configuration of a polypeptide chain, and is believed to be NP-hard, because finding the global energy minimum with all possible interactions is not at all easy. The rephrased problem of structural design may not be that hard - the local orientation of a building block can be fixed by its interactions with its neighbours; it is enough to have a locally stable or metastable configuration and not necessarily a global energy minimum. (Diamond is structurally the strongest material, but it is energetically metastable.) Also, there are many ways a folded chain can cover a three-dimensional shape, and quite likely there is a lot of flexibility in choosing the sequence of amino acids without substantially altering the structure of the protein.

The fundamental operations needed for processing structural information are translation and rotation. I have shown that carbon and its tetrahedral geometry provide the simplest discretization of these operations. For the construction of proteins as folded chains, the polypeptide chain is the simplest back-bone containing rigid segments alternating with flexible joints. To fold this back-bone into arbitrary shapes on a diamond lattice requires 10 basic operations. The amino acids somehow implement these operations by interactions amongst their side chain $R$-groups.

I have pointed out that the division of the 20 amino acids, by aminoacyl-tRNA synthetases, into two classes of 10 each has structural significance. Every $R$-group property is equally divided between the two classes, such that the shorter side chains are in class II and the longer ones in class I. This is a new observation. Combining this fact with the number of discrete operations required to fold a polypeptide chain, and the result that two yes/no quantum queries can distinguish 10 items, I have proposed that the present triplet genetic code was preceded by a primitive doublet one. How the doublet code was converted to a triplet one is a matter of conjecture, and I have outlined one possible scenario.

Knowing the solution selected by evolution has no doubt guided my logic. Still unraveling the optimization criteria involved in the design of molecules of life is a thrilling exercise. It should be kept in mind that evolution has discovered its optimal parameters, not by logical 
deduction, but by trial and error experiments (of course using the available means). For that reason the chosen parameters are not always perfect. On the other hand, evolution has had plenty of time for experimentation, something which we do not, and cannot, have. As a result, though evolution is not perfect in finding its criteria, it is impressive to say the least!

A shortcoming of my analysis is that the role played by water in protein folding is totally ignored. Water molecules do not provide just a uniform background inside the cells; they fit in tetrahedral geometry nicely, and are therefore well-suited to fill up empty grooves and cavities of proteins. Any explanation of interactions amongst the side chain $R$-groups of amino acids must include how the $R$-groups interact with water. This unfinished exercise is for the future.

The ideas discussed in this work have potential applications. Molecular dynamics simulations of protein folding have typically been carried out in the continuous three-dimensional space. The discrete information theory language can speed up these simulations, by first folding the polypeptide chain on a diamond lattice and then switching to the continuous space to fine tune the actual atomic positions. In recent years, lattice models have been used for the analysis of polypeptide folds. But they have mostly used cubic or triangular lattices and restricted amino acid properties to a binary hydrophobic/ hydrophilic choice (Hart and Istrail 1997; Agarwala et al 1997). These models have had only a limited success in understanding the energy landscape of the protein folding process. My analysis suggests that the use of a diamond lattice and more details of amino acid properties will bring the lattice models closer to reality. Of course, if the preferences of amino acid sequences for certain lattice positions are known (e.g. from the analysis of protein structure databases), that can simplify the simulations further.

From the view-point of nanotechnology, construction of desired molecular structures by chains that know how to fold is a conceptual shift from the conventional approaches that use external sources (either to carve the shapes or to assemble individual building blocks). To be able to do that, the folding code of amino acid sequences must be deciphered; wide ranging applications are obvious. One can also consider the simpler problem of constructing two dimensional patterns by folding chains made of flat building blocks. Such an approach will be a contrast to the standard techniques of lithography, but it requires first figuring out the appropriate two-dimensional building blocks and processes that can join them together in chains. Carbon rings and the geometry of graphite sheets will no doubt play a central role as the optimal ingradients in such an exercise.

Finally, I want to contrast different information processing paradigms. Electronic computation uses physical building blocks and operations based on real variables. Quantum computation extends the building blocks and operations to the complex numbers. Structural information processing goes still one step further, to the noncommutative algebra of quaternions. Systematic analysis of structural information processing has a long way to go. Yet in a sense, it came first - proteins arose before genes, nervous signals, spoken and written languages, number systems and computers. After all, the word "protein" derives from the Greek "protos" meaning "first" or "foremost".

\section{Acknowledgements}

I am grateful to C Ramakrishnan and M A Viswamitra for useful discussions regarding protein structure and folding. The Ramachandran map in figure 1 was kindly provided by $\mathrm{C}$ Ramakrishnan.

\section{Appendix: Properties of proteins}

Many careful experiments have been performed over the years to study various properties of proteins. Here I list some of the important features they have revealed, as a quick reference (Lehninger et al 1993; Creighton 1992; Fersht 1999):

- Proteins are synthesized by ribosomes by joining amino acids together as a linear chain. The linear chain gradually folds into the three-dimensional shape unique to every protein. The folding occurs by rigid body transformations of the bonds - deformations such as stretching/ shrinking/bending of bonds are insignificant.

- Proteins carry out their tasks by binding to various molecules. The binding is highly specific, very much like a lock and key arrangement. Structurally stable features, precisely located on the protein surface, are necessary for this purpose. When a protein fails to fold into its proper shape due to some error, it cannot carry out its task and that gives rise to a disease. (Sickle-cell anaemia was the first genetic disease to be understood in this manner. A single mutation substitutes glutamic acid with valine at the sixth position in the amino acid sequence. The resultant defective haemoglobin does not fold correctly and is unable to carry out its function properly.)

- The sequence of amino acids encodes the structural information of a protein. Figure 3 shows the structure of individual amino acids. Proteins may include other important components, e.g. iron in haemoglobin, but the role of these other components is essentially chemical and not structural.

- The sequence of amino acids is obtained by translation from the sequence of nucleotide bases in DNA. This 
translation is necessary because the two languages serve two different purposes, and the purposes decide the physical components for their realizations. According to the cell's need, proteins are synthesized, transported to appropriate locations to participate in biochemical reactions, and degraded at the end. The three-dimensional shape of the protein plays a critical part in its reactivity. The double helical structure of DNA, with nucleotide bases hidden inside, protects the one-dimensional information until it is required. DNA replication is also much less error-prone than protein synthesis.

- The physical separation between consecutive amino acids in polypeptide chains and consecutive nucleotide bases in DNA is about the same, approximately $3.5 \AA$. It is not direct stereochemistry, therefore, which is responsible for three nucleotide bases being mapped to one amino acid in the genetic code. The non-overlapping triplet code is likely to have arisen from the need to have a sufficient number of amino acids as the required building blocks for the three-dimensional protein structure. Living organisms had to then set up the complex machinery, involving tRNA as adapters connecting nucleotide bases and amino acids, to carry out the task of translation.

- Correct translation is ensured by the bilingual aminoacyl-tRNA synthetases that attach amino acids to tRNA molecules with appropriate anticodons. There may be several anticodons which map to a particular amino acid, but there is only one aminoacyl-tRNA synthetase per amino acid which carries out the many-to-one mapping. Once the tRNA molecules are properly charged with amino acids, the ribosomes match the anticodons of tRNA with codons of mRNA and construct the polypeptide chain.

- Proteins often have to cross membranes and cell walls after their synthesis, since they often have to carry out their tasks at locations other than their place of synthesis. Membranes and cell walls cannot afford to have big holes (otherwise many molecules would leak), and that provides an important reason why proteins are folded chains. During translocation, proteins unfold to their chain form, cross the barrier through a small hole and then fold again into their native three-dimensional form.

- The three-dimensional protein structure specified by the sequence of amino acids is essentially unique. Small proteins fold on their own, but many large proteins require help of molecular chaperons to fold. Globular proteins that fold on their own can be melted by heat, and they regain their native form upon cooling.

- Carbon and nitrogen atoms, joined by strong covalent bonds, form the back-bone of the polypeptide chain. In this chain rigid peptide bonds alternate with rotatable bonds of $\mathrm{C}_{\alpha}$ atoms. The $\mathrm{C}-\mathrm{N}$ peptide bonds have a double bond character; the nitrogen atom carries a positive charge making its electronic behaviour similar to the tetravalent carbon atom.

- Different amino acids are distinguished from each other by their $R$-groups, which are side chains attached to the $\mathrm{C}_{\alpha}$ atoms. Amino acid $R$-groups are of various types: polar, non-polar, aromatic, positively and negatively charged. The interactions of these $R$-groups with each other and with the ambient water molecules fix the orientations of the rotatable $\mathrm{C}_{\alpha}$ bonds. These interactions are weak, and easily influenced by the $\mathrm{pH}$ of the ambient liquid and the temperature.

- Atoms in proteins are quite densely packed. In terms of the van der Waals atomic size, packing fraction for proteins is in the range $0.70-0.78$, compared to 0.74 for closest packing of identical spheres. The packing fraction of a diamond lattice is only $0 \cdot 34$, and the side groups of a polypeptide chain folded along a diamond lattice fill up the empty spaces. Even then the packing density is high along the chain, while the amino acid side groups are somewhat loosely packed. Small cavities are filled up by water molecules, which fit into tetrahedral geometry nicely. - The polypeptide chain is synthesized in the fully extended form, corresponding to $\phi=180^{\circ}=\psi$ and "trans" configuration. Certain domains of proteins start folding as soon as they are synthesized, indicating that at least some of the folding rules are local.

- The folding process occurs in stages. Local domains fold first, essentially due to weak bonds (hydrogen and van der Waals). This process is dominated by local transformations, i.e. proper rotations of bonds of $\mathrm{C}_{\alpha}$ atoms, and forms well-known structures such as $\alpha$-helices and $\beta$ sheets. In the next stage, already folded domains get linked by long-distance connections, e.g. disulfide bonds. In the final stage, various separately assembled structures, polypeptide chains and chemical groups, join together.

- Regular structures like $\alpha$-helices and $\beta$-sheets are largely determined by the properties of the polypeptide back-bone, with a lot of freedom in choice of amino acid $R$-groups. It is the irregular twists and turns of the chain which critically depend on the interactions of the amino acid $R$-groups. Several different type of interactions exist (electric charges, dipoles, hydrogen bonds, rings, bifurcation and bulk of side chains etc.) to achieve all possible shapes.

- In reality, proteins fold rather rapidly. The folded chain is a self-avoiding walk in three-dimensional space. Such a walk can get stuck for topological reasons, or it may need global criteria to complete its task (travelling salesman type of problems are NP-hard with just local rules). An easy escape is to complete the task with multiple walks, i.e. start a new walk when the previous one gets stuck. Indeed many proteins are made of not a single polypeptide chain, but several polypeptide chains entangled together. Large protein structures are often made of 
polypeptide units arranged in regular patterns (e.g. fibroin, keratin, collagen, virus coats etc.).

- Figure 2c shows the rotation angles around single bonds in a polypeptide chain. Because of steric conflict between various atoms, not all the values of angles occur in a polypeptide chain. The Ramachandran map $(\phi-\psi$ angular distribution) in figure 1 displays the orientations available to the amino acids. Experimental data for polypeptide chains follow the constraints of the Ramachandran map quite well; in fact, the map is often used as a filter for models when the experimental data are not accurate enough. The side chain angles $(\chi)$ also have preferred orientations which are separated by $120^{\circ}$.

- Structural roles played by some of the amino acids are well-known. Glycine with no side chain and no chiral centre is the most flexible. Proline with its rigid ring and trans-cis transformation plays an important role in forming sharp bends. Cysteine connects far separated regions of the polypeptide chain by strong disulfide bonds, helping the folded chain retain its shape.

- Ordinary chemical reactions produce a mixture of Dtype and L-type molecules, but biological processes utilize molecules of only L-type chirality. The smallest amino acid glycine is achiral, the next smallest alanine is once in a while found in D-type configuration, while the rest are always in L-type configuration. There exist racemase enzymes which can flip chirality of amino acids. Most of the time they convert D-type amino acids to L-type for use in protein synthesis. After a cell dies, its molecules gradually revert to a mixture of D- and Ltypes. The proportion of D- and L-type molecules in a dead cell can indeed be used to figure out how long back the cell died.

\section{References}

Agarwala R, Batzoglou S, Dančik V, Decatur S E, Hannenhalli S, Farach M, Muthukrishnan S and Skiena S 1997 Local rules for protein folding on a triangular lattice and generalized hydrophobicity in the HP model; J. Comput. Biol. 4 275-296

Arnez J G and Moras D 1997 Structural and functional considerations of the aminoacylation reaction; Trends Biochem. Sci. 22 211-216

Creighton T E (ed.) 1992 Protein folding (New York: W H Freeman)

Crick F H C 1966 Codon-anticodon pairing: The wobble hypothesis; J. Mol. Biol. 19 548-555

Crick F H C 1968 The origin of the genetic code; J. Mol. Biol. 38 367-379

Eriani G, Delarue M, Poch O, Gangloff J and Moras D 1990 Partition of tRNA synthetases into two classes based on mutually exclusive sets of sequence motifs; Nature (London) 347 203-206

Fersht A 1999 Structure and mechanism in protein science: A guide to enzyme catalysis and protein folding (New York: W H Freeman)

Grover L 1997 Quantum mechanics helps in searching for a needle in a haystack; Phys. Rev. Lett. 79 325-328 [quant$\mathrm{ph} / 9706033$ ]

Hart W E and Istrail S 1997 Lattice and off-lattice side chain models of protein folding: Linear time structure prediction better than $86 \%$ of optimal; J. Comput. Biol. 4 241259

Ikehara K 2002 Origins of gene, genetic code, protein and life: Comprehensive view of life systems from a GNCSNS primitive genetic code hypothesis; J. Biosci. 27 165186

Kolaskar A S and Ramabrahmam V 1982 Obligatory amino acids in primitive proteins; Biosystems 15 105-109

Lehninger A L, Nelson D L and Cox M M 1993 Principles of biochemistry second edition (USA: Worth Publishers)

Lewin B 2000 Genes VII (Oxford: Oxford University Press)

Patel A 2000 Quantum algorithms and the genetic code; Proc. Winter Institute on Foundations of Quantum Theory and Quantum Optics (Calcutta); Pramāna-J. Phys. 56 367-381 [quant-ph/0002037]

Patel A 2001 Quantum database search can do without sorting; Phys. Rev. A 64034303 [quant-ph/0012149]

Ramachandran G N, Ramakrishnan C and Sasisekharan V 1963 Stereochemistry of polypeptide chain configurations; J. Mol. Biol. 7 95-99

MS received 1 April 2002; accepted 2 May 2002

Corresponding editor: VIDYANAND NANJUNDIAH 